\title{
The global electroweak fit at NNLO and prospects for the LHC and ILC
}

\author{
The Gfitter Group, \\ M. Baak ${ }^{1}$, J. Cúth ${ }^{2}$, J. Haller ${ }^{3}$, A. Hoecker ${ }^{1}$, R. Kogler ${ }^{3, a}$, K. Mönig ${ }^{4}$, M. Schott ${ }^{2}$, J. Stelzer ${ }^{5}$ \\ ${ }^{1}$ CERN, Geneva, Switzerland \\ 2 Institut für Physik, Universität Mainz, Mainz, Germany \\ ${ }^{3}$ Institut für Experimentalphysik, Universität Hamburg, Hamburg, Germany \\ ${ }^{4}$ DESY, Hamburg and Zeuthen, Germany \\ ${ }^{5}$ Department of Physics and Astronomy, Michigan State University, East Lansing, USA
}

Received: 17 July 2014 / Accepted: 27 August 2014 / Published online: 16 September 2014

(C) The Author(s) 2014. This article is published with open access at Springerlink.com

\begin{abstract}
For a long time, global fits of the electroweak sector of the standard model (SM) have been used to exploit measurements of electroweak precision observables at lepton colliders (LEP, SLC), together with measurements at hadron colliders (Tevatron, LHC) and accurate theoretical predictions at multi-loop level, to constrain free parameters of the SM, such as the Higgs and top masses. Today, all fundamental SM parameters entering these fits are experimentally determined, including information on the Higgs couplings, and the global fits are used as powerful tools to assess the validity of the theory and to constrain scenarios for new physics. Future measurements at the Large Hadron Collider (LHC) and the International Linear Collider (ILC) promise to improve the experimental precision of key observables used in the fits. This paper presents updated electroweak fit results using the latest NNLO theoretical predictions and prospects for the LHC and ILC. The impact of experimental and theoretical uncertainties is analysed in detail. We compare constraints from the electroweak fit on the Higgs couplings with direct LHC measurements, and we examine present and future prospects of these constraints using a model with modified couplings of the Higgs boson to fermions and bosons.
\end{abstract}

\section{Introduction}

Global fits of the standard model (SM) have traditionally combined electroweak precision observables with accurate theoretical predictions to constrain the top-quark and Higgs boson masses [1-5]. The discovery of a scalar boson at the Large Hadron Collider (LHC) [6,7], with mass $M_{H}$ around $125 \mathrm{GeV}$, provides an impressive confirmation of the light Higgs prediction derived from these fits. Assuming the new

\footnotetext{
a e-mail: roman.kogler@physik.uni-hamburg.de
}

boson to be the SM Higgs boson and inserting the measured mass into the fit overconstrains the electroweak sector of the SM. Key electroweak observables such as the $W$ boson mass, $M_{W}$, and the effective weak mixing angle for charged and neutral leptons and light quarks, $\sin ^{2} \theta_{\text {eff }}^{f}$, can thus be predicted with a precision exceeding that of the direct measurements [8]. These observables become sensitive probes of new physics [9] limited in part by the accuracy of the theoretical calculations.

Recently, full fermionic two-loop calculations have become available for the partial widths and branching ratios of the $Z$ boson [10]. These new calculations improve the theoretical precision and also allow for a more meaningful estimate of the theoretical uncertainties due to missing higher perturbative orders. In this paper we present an update of the global electroweak fit performed at the two-loop level, ${ }^{1}$ including a detailed assessment of the impact of the remaining theoretical uncertainties.

Measurements performed at future colliders will increase the experimental precision of these and other electroweak observables, such as the top-quark mass, $m_{t}$. The coming years should also lead to progress in the calculation of multi-loop corrections to these observables, as well as to an improved determination of the hadronic contribution to the fine-structure constant evaluated at the $Z$ boson mass scale, $\Delta \alpha_{\text {had }}\left(M_{Z}^{2}\right)$.

In this paper, the latest results of the global electroweak fit are compared with the expectations for the Phase- $1 \mathrm{LHC}^{2}$ and

\footnotetext{
1 The decay width of the $W$ boson, $\Gamma_{W}$, is only known to one-loop precision. However, this measurement being of insufficient precision has a negligible impact on the result of the electroweak fit.

2 This corresponds to a scenario with $\int L \mathrm{~d} t=300 \mathrm{fb}^{-1}$ at $\sqrt{s}=$ $14 \mathrm{TeV}$, before the high luminosity upgrade.
} 
the International Linear Collider (ILC) with GigaZ option, ${ }^{3}$ henceforth denoted ILC/GigaZ [11]. For each scenario we analyse the impact of the assigned experimental and theoretical uncertainties. By exploiting contributions from radiative corrections, the global electroweak fit is also used to determine the couplings of the Higgs boson to gauge bosons using the formalism of the $S, T, U$ parameters. We combine the constraints on the Higgs couplings in a popular benchmark model with LHC measurements of the signal strength in various channels. We also study the prospects for these constraints.

The paper is organised as follows. An update of the global electroweak fit including the recent theoretical improvements is presented in Sect. 2. Section 3 discusses the extrapolated uncertainties of key input observables for the LHC and ILC/GigaZ scenarios, the fit prospects and a detailed analysis of the impact of all sources of systematic uncertainties. The status and prospects for the determinations of Higgs couplings from the electroweak fit are reported in Sect. 4.

\section{Update of the global electroweak fit}

In the following we present an update of the global electroweak fit at the $Z$-mass scale. The relevant observables, the data treatment and statistical framework are described in Refs. [3,4]. We use the recent calculations of the $Z$ boson partial widths and branching ratios at the electroweak twoloop order [10]. These provide for the first time a consistent set of calculations at next-to-next-to leading order (NNLO) for all relevant input observables, together with the two-loop calculations of the $W$ mass and the effective weak mixing angle.

\subsection{SM predictions and theoretical uncertainties}

The following theoretical predictions of the SM observables are used.

- The effective weak mixing angle, $\sin ^{2} \theta_{\text {eff }}^{f}$, has been calculated using corrections up to the full two-loop order $\mathcal{O}\left(\alpha \alpha_{s}\right)$ and $\mathcal{O}\left(\alpha^{2}\right)[13,14]$. In addition, partial threeloop and four-loop terms have been included at order $\mathcal{O}\left(\alpha \alpha_{s}^{2}\right)[15-17], \mathcal{O}\left(\alpha_{t}^{2} \alpha_{s}\right), \mathcal{O}\left(\alpha_{t}^{3}\right)[18,19]$ and $\mathcal{O}\left(\alpha_{t} \alpha_{s}^{3}\right)$

\footnotetext{
${ }^{3}$ GigaZ: the operation of the ILC at lower energies like the $Z$ pole or the $W W$ threshold allows for high-statistics precision measurements of several electroweak observables. At the $Z$ pole the physics at LEP and SLC can be revisited with the data collected during a few days. Several billion $Z$ bosons can be produced within a few months [11]. In comparison: in the seven years that LEP operated at the $Z$ peak it produced around 17 million $Z$ bosons in its four interaction points; SLD studied about 600 thousand $Z$ bosons produced with a polarised beam [12].
}

[20-22], where $\alpha_{t}=\alpha m_{t}^{2}$. These calculations have been included in the parametrisation provided in $[13,14]$. For bottom quarks the calculation from [23] is used, which includes corrections of the same order together with additional vertex corrections from top-quark propagators.

- The mass of the $W$ boson, $M_{W}$, has been calculated to the same orders of electroweak and QCD corrections as $\sin ^{2} \theta_{\text {eff }}^{f}$. We use the parametrisation of the full two-loop result [24]. New in this paper is the inclusion of fourloop QCD corrections $\mathcal{O}\left(\alpha_{t} \alpha_{s}^{3}\right)$ [20-22], which result in a shift of the predicted $M_{W}$ by about $-2.2 \mathrm{MeV}$ in the on-shell renormalisation scheme for $m_{t}$. The exact value of the shift depends on the parameter settings used.

- Full fermionic two-loop corrections $\mathcal{O}\left(\alpha^{2}\right)$ for the partial widths and branching ratios of the $Z$ boson have recently become available [10]. ${ }^{4}$ The parametrisation formulae provided include also higher-order terms to match the perturbative order of the calculations of $\sin ^{2} \theta_{\mathrm{eff}}^{f}$ and $M_{W}$. These calculations amend previous two-loop predictions of the total $Z$ width, $\Gamma_{Z}$, the hadronic peak cross section, $\sigma_{\text {had }}^{0}[25]$ and the partial decay width of the $Z$ boson into $b \bar{b}, R_{b}^{0}$ [26].

- The dominant contributions from final-state QED and QCD radiation are included in the calculations through factorisable radiator functions $\mathcal{R}_{V, A}$, which are known up to $\mathcal{O}\left(\alpha_{s}^{4}\right)$ for massless final-state quarks, $\mathcal{O}\left(\alpha_{s}^{3}\right)$ for massive quarks [27-29] and $\mathcal{O}\left(\alpha^{2}\right)$ for contributions with closed fermion loops [30]. Non-factorisable vertex contributions of order $\mathcal{O}\left(\alpha \alpha_{s}\right)$ [31,32] are also accounted for.

- The width of the $W$ boson, $\Gamma_{W}$, is known up to one electroweak loop order. We use the parametrisation given in [33], which is sufficient given the experimental precision.

In summary, the changes with respect to our previous publication [8] are the addition of the $\mathcal{O}\left(\alpha_{t} \alpha_{s}^{3}\right)$ QCD correction to $M_{W}$ and the two-loop calculations of the partial $Z$ widths. The latter calculations also yield an updated result for $R_{b}^{0}$, including non-factorisable $\mathcal{O}\left(\alpha \alpha_{s}\right)$ and $\mathcal{O}\left(\alpha_{s}^{4}\right)$ corrections. For the calculations of the SM predictions we use the computer code employed previously [8], with the corresponding updates.

The theoretical uncertainties from unknown higher-order contributions have been estimated by assuming that the perturbation series follow a geometric growth [10,14,24]. The resulting uncertainties for $M_{W}, \sin ^{2} \theta_{\text {eff }}^{f}, \sigma_{\text {had }}^{0}$ and all decay widths, $\Gamma_{f}$, for the decay $Z \rightarrow f \bar{f}$, are listed in Table 1 .

\footnotetext{
4 These calculations do not include diagrams with closed boson loops at two-loop order. These are expected to give small corrections compared to diagrams with closed fermion loops and are therefore only considered in the estimate of the theoretical uncertainty.
} 
Table 1 Theory uncertainties taken into account in the global electroweak fit. See text for details

\begin{tabular}{llll}
\hline$\delta_{\text {theo }} M_{W}$ & $4 \mathrm{MeV}$ & $\delta_{\text {theo }} \Gamma_{u, c}$ & $0.12 \mathrm{MeV}$ \\
$\delta_{\text {theo }} \sin ^{2} \theta_{\text {eff }}^{f}$ & $4.7 \times 10^{-5}$ & $\delta_{\text {theo }} \Gamma_{b}$ & $0.21 \mathrm{MeV}$ \\
$\delta_{\text {theo }} \Gamma_{e, \mu, \tau}$ & $0.012 \mathrm{MeV}$ & $\delta_{\text {theo }} \sigma_{\text {had }}^{0}$ & $6 \mathrm{pb}$ \\
$\delta_{\text {theo }} \Gamma_{v}$ & $0.014 \mathrm{MeV}$ & $\delta_{\text {theo }} \mathcal{R}_{V, A}$ & $\sim \mathcal{O}\left(\alpha_{s}^{4}\right)$ \\
$\delta_{\text {theo }} \Gamma_{d, s}$ & $0.09 \mathrm{MeV}$ & $\delta_{\text {theo }} m_{t}$ & $0.5 \mathrm{GeV}$ \\
\hline
\end{tabular}

For $M_{W}$ and $\sin ^{2} \theta_{\text {eff }}^{f}$ they arise from three dominant sources of unknown higher-order corrections: $\mathcal{O}\left(\alpha^{2} \alpha_{S}\right)$ terms beyond the known contribution of $\mathcal{O}\left(G_{F}^{2} \alpha_{S} m_{t}^{4}\right), \mathcal{O}\left(\alpha^{3}\right)$ electroweak three-loop corrections and $\mathcal{O}\left(\alpha_{S}^{3}\right)$ QCD terms. Summing quadratically the relevant uncertainty estimates amounts to the overall theoretical uncertainties $\delta_{\text {theo }} M_{W}=$ $4 \mathrm{MeV}$ [24] and $\delta_{\text {theo }} \sin ^{2} \theta_{\text {eff }}^{f}=4.7 \times 10^{-5}$ [14].

The leading theoretical uncertainties on the predicted $Z$ decay widths and $\sigma_{\text {had }}^{0}$ come from missing two-loop electroweak bosonic $\mathcal{O}\left(\alpha^{2}\right)$ contributions, three-loop terms of order $\mathcal{O}\left(\alpha^{3}\right), \mathcal{O}\left(\alpha^{2} \alpha_{S}\right)$ and $\mathcal{O}\left(\alpha \alpha_{S}^{2}\right)$ and $\mathcal{O}\left(\alpha \alpha_{S}^{3}\right)$ corrections beyond the leading $m_{t}^{n}$ terms. The resulting uncertainties $\delta_{\text {theo }} \Gamma_{f}$ are between 0.012 and $0.21 \mathrm{MeV}$ (see Table 1). The theoretical uncertainty $\delta_{\text {theo }} \sigma_{\text {had }}^{0}$ amounts to $6 \mathrm{pb}$.

Uncertainties due to unknown higher-order contributions to the radiator functions $\mathcal{R}_{V, A}$ have been estimated by varying the $\mathcal{O}\left(\alpha_{s}^{4}\right)$ terms for the massless- and massive-quark contributions by factors of 0 to 2 . The uncertainty due to the singlet vector contribution was found to be negligible.

We assign an additional theoretical uncertainty to the value of $m_{t}$ from hadron collider measurements due to the ambiguity in the kinematic top-mass definition [34-37], the colour structure of the fragmentation process $[38,39]$ and the perturbative relation between pole and $\overline{\mathrm{MS}}$ mass currently known to three-loop order $[40,41]$. The first uncertainty is difficult to assess. Estimates range from 0.25 to $0.9 \mathrm{GeV}$ or higher [37,42]. Systematic effects on $m_{t}$ due to mis-modelling of the colour reconnection in the fragmentation process, initialand final-state radiation and the kinematics of the $b$-quark are partly considered as uncertainties by the experiments. They were also studied in a dedicated measurement by CMS [43] where no significant trends between the measurements under different conditions were observed. Finally, estimates of the missing higher-order perturbative correction to the relation between the pole and $\overline{\mathrm{MS}}$ top-mass range from 0.2 to $0.3 \mathrm{GeV}$ uncertainty [44]. The nominal value of the combined $m_{t}$ uncertainty is set here to $0.5 \mathrm{GeV}$. The impact of this uncertainty is studied below for values between 0 and $1.5 \mathrm{GeV}$.

Our previous publications $[3,4,8]$ employed the $R$ fit scheme, characterised by uniform likelihoods for the two theoretical nuisance parameters $\delta_{\text {theo }} M_{W}$ and $\delta_{\text {theo }} \sin ^{2} \theta_{\text {eff }}^{f}$ used. It corresponds to a linear addition of theoretical and experimental uncertainties. In this analysis, with the ten theoretical nuisance parameters listed in Table 1, we use Gaussian constraints to stabilise the fit convergence. The Gaussian nuisance parameter treatment modifies how the theoretical uncertainties impact the fit. The resulting $\chi^{2}$ curve for a given fit parameter is narrower around its minimum value than that obtained with the $R$ fit treatment, while it is broader for large deviations. This property should be kept in mind when interpreting the results.

\subsection{Experimental input}

A detailed list of all the observables, their values and uncertainties used in the fit, is given in the first two columns of Table 2. The input data to the fit consist of measurements at the $Z$ pole by the LEP and SLD collaborations [12], the world average values for the running quark masses [45], of $M_{W}$ and $\Gamma_{W}$ [45] and an up-to-date determination of the fivequark hadronic vacuum polarisation contribution to $\alpha\left(M_{Z}^{2}\right)$, $\Delta \alpha_{\text {had }}^{(5)}\left(M_{Z}^{2}\right)$ [46]. For the mass of the top quark we use the latest average from the direct measurements by the LHC and Tevatron experiments [47] with the additional $0.5 \mathrm{GeV}$ theoretical uncertainty as discussed above. The mass of the Higgs boson $M_{H}=125.14 \pm 0.24 \mathrm{GeV}$ is computed from the weighted average of the new results by ATLAS [48] $(125.4 \pm 0.4 \mathrm{GeV})$ and CMS [49] (125.0 $\pm 0.3 \mathrm{GeV})$, where correlations between the measurements are ignored.

\subsection{Results of the SM fit}

The fit of the electroweak theory to all input data from Table 2, including the theoretical uncertainties from Table 1, converges at a global minimum value of $\chi_{\min }^{2}=17.8$, obtained for 14 degrees of freedom. Using pseudo experiments and the statistical method described in [3] we find a $p$-value for the $\mathrm{SM}$ to describe the data of 0.21 (corresponding to $0.8 \sigma$ onesided significance). The improved goodness-of-fit compared to earlier results [8] comes mostly from the corrected calculation of $R_{b}^{0}$ [26], which decreases the previously reported discrepancy of $R_{b}^{0}$ between the global fit and the measurement from a pull value of $-2.4 \sigma$ down to $-0.8 \sigma$ (consistent with the one-loop calculation of $R_{b}^{0}$ ). The impact of this change on the other fit parameters is small. The new two-loop calculations of the $Z$ partial widths decrease the value of $\chi_{\min }^{2}$ by 0.2 , whereas the $\mathcal{O}\left(\alpha_{t} \alpha_{s}^{3}\right)$ four-loop QCD corrections to $M_{W}$ increase $\chi_{\min }^{2}$ by 0.4 units.

The results of the full fit for each fit parameter and observable are given in the fourth column of Table 2, together with the uncertainties estimated from their $\Delta \chi^{2}=1$ profiles. The fifth column in Table 2 gives the results obtained without using in the fit the experimental measurement corresponding to that row. A more detailed discussion of these indi- 
Table 2 Input values and fit results for the observables used in the global electroweak fit. The first and second columns list, respectively, the observables/parameters used in the fit and their experimental values or phenomenological estimates (see text for references). The third column indicates whether a parameter is floating in the fit. The fourth column quotes the results of the fit including all experimental data. In the fifth column the fit results are given without using the corresponding experimental or phenomenological estimate in the given row (indirect determination). The last column shows for illustration the result using the same fit setup as in the fifth column, but ignoring all theoretical uncertainties. The nuisance parameters that are used to parameterise theoretical uncertainties are given in Table 1

\begin{tabular}{|c|c|c|c|c|c|}
\hline Parameter & Input value & $\begin{array}{l}\text { Free } \\
\text { in fit }\end{array}$ & Fit result & w/o exp. input in line & $\begin{array}{l}\text { w/o exp. input in line, } \\
\text { no theo. unc }\end{array}$ \\
\hline$M_{H}[\mathrm{GeV}]^{\mathrm{a}}$ & $125.14 \pm 0.24$ & Yes & $125.14 \pm 0.24$ & $93_{-21}^{+25}$ & $93_{-20}^{+24}$ \\
\hline$M_{W}[\mathrm{GeV}]$ & $80.385 \pm 0.015$ & - & $80.364 \pm 0.007$ & $80.358 \pm 0.008$ & $80.358 \pm 0.006$ \\
\hline$\Gamma_{W}[\mathrm{GeV}]$ & $2.085 \pm 0.042$ & - & $2.091 \pm 0.001$ & $2.091 \pm 0.001$ & $2.091 \pm 0.001$ \\
\hline$M_{Z}[\mathrm{GeV}]$ & $91.1875 \pm 0.0021$ & Yes & $91.1880 \pm 0.0021$ & $91.200 \pm 0.011$ & $91.2000 \pm 0.010$ \\
\hline$\Gamma_{Z}[\mathrm{GeV}]$ & $2.4952 \pm 0.0023$ & - & $2.4950 \pm 0.0014$ & $2.4946 \pm 0.0016$ & $2.4945 \pm 0.0016$ \\
\hline$\sigma_{\text {had }}^{0}[\mathrm{nb}]$ & $41.540 \pm 0.037$ & - & $41.484 \pm 0.015$ & $41.475 \pm 0.016$ & $41.474 \pm 0.015$ \\
\hline$R_{\ell}^{0}$ & $20.767 \pm 0.025$ & - & $20.743 \pm 0.017$ & $20.722 \pm 0.026$ & $20.721 \pm 0.026$ \\
\hline$A_{\mathrm{FB}}^{0, \ell}$ & $0.0171 \pm 0.0010$ & - & $0.01626 \pm 0.0001$ & $0.01625 \pm 0.0001$ & $0.01625 \pm 0.0001$ \\
\hline$A_{\ell} \mathrm{b}$ & $0.1499 \pm 0.0018$ & - & $0.1472 \pm 0.0005$ & $0.1472 \pm 0.0005$ & $0.1472 \pm 0.0004$ \\
\hline $\sin ^{2} \theta_{\mathrm{eff}}^{\ell}\left(Q_{\mathrm{FB}}\right)$ & $0.2324 \pm 0.0012$ & - & $0.23150 \pm 0.00006$ & $0.23149 \pm 0.00007$ & $0.23150 \pm 0.00005$ \\
\hline$A_{c}$ & $0.670 \pm 0.027$ & - & $0.6680 \pm 0.00022$ & $0.6680 \pm 0.00022$ & $0.6680 \pm 0.00016$ \\
\hline$A_{b}$ & $0.923 \pm 0.020$ & - & $0.93463 \pm 0.00004$ & $0.93463 \pm 0.00004$ & $0.93463 \pm 0.00003$ \\
\hline$A_{\mathrm{FB}}^{0, c}$ & $0.0707 \pm 0.0035$ & - & $0.0738 \pm 0.0003$ & $0.0738 \pm 0.0003$ & $0.0738 \pm 0.0002$ \\
\hline$A_{\mathrm{FB}}^{0, b}$ & $0.0992 \pm 0.0016$ & - & $0.1032 \pm 0.0004$ & $0.1034 \pm 0.0004$ & $0.1033 \pm 0.0003$ \\
\hline$R_{c}^{0}$ & $0.1721 \pm 0.0030$ & - & $0.17226_{-0.00008}^{+0.00009}$ & $0.17226 \pm 0.00008$ & $0.17226 \pm 0.00006$ \\
\hline$R_{b}^{0}$ & $0.21629 \pm 0.00066$ & - & $0.21578 \pm 0.00011$ & $0.21577 \pm 0.00011$ & $0.21577 \pm 0.00004$ \\
\hline $\bar{m}_{c}[\mathrm{GeV}]$ & $1.27_{-0.11}^{+0.07}$ & Yes & $1.27_{-0.11}^{+0.07}$ & - & - \\
\hline $\bar{m}_{b}[\mathrm{GeV}]$ & $4.20_{-0.07}^{+0.17}$ & Yes & $4.20_{-0.07}^{+0.17}$ & - & - \\
\hline$m_{t}[\mathrm{GeV}]$ & $173.34 \pm 0.76$ & Yes & $173.81 \pm 0.85$ & $177.0_{-2.4}^{+2.3 \mathrm{c}}$ & $177.0 \pm 2.3^{\mathrm{c}}$ \\
\hline$\Delta \alpha_{\text {had }}^{(5)}\left(M_{Z}^{2}\right)^{\mathrm{d}, \mathrm{e}}$ & $2757 \pm 10$ & Yes & $2756 \pm 10$ & $2723 \pm 44$ & $2722 \pm 42$ \\
\hline$\alpha_{s}\left(M_{Z}^{2}\right)$ & - & Yes & $0.1196 \pm 0.0030$ & $0.1196 \pm 0.0030$ & $0.1196 \pm 0.0028$ \\
\hline
\end{tabular}

a Average of the ATLAS [48] and CMS [49] measurements assuming no correlation of the systematic uncertainties

b Average of the LEP and SLD $A_{\ell}$ measurements [12], used as two measurements in the fit

${ }^{c}$ The theoretical top-mass uncertainty of $0.5 \mathrm{GeV}$ is excluded

d In units of $10^{-5}$

e Rescaled due to $\alpha_{s}$ dependence

rect determinations for several key observables is given in Sect. 3.2. The last column in Table 2 corresponds to the fits of the previous column but ignoring, for the purpose of illustration, all theoretical uncertainties. In this case the global fit converges at a slightly increased minimum value of $\chi_{\min }^{2} /$ ndf $=18.2 / 14$.

The result of the fit is summarised in Fig. 1. The plot on the left shows a comparison of the global fit results (fourth column of Table 2) with the direct measurements (first column of Table 2) in units of the measurement uncertainty. Also shown is the impact of the two-loop result for the $Z$ partial widths and the $\mathcal{O}\left(\alpha_{t} \alpha_{s}^{3}\right)$ correction to $M_{W}$, compared to the calculations previously used ${ }^{5}[8]$. The right-hand panel of

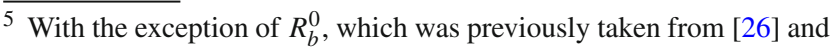
was later corrected. For this comparison the one-loop result [33] is used.
}

Fig. 1 displays the comparison of both the global fit result and the direct measurements with the indirect determination (fifth column of Table 2) for each observable in units of the total uncertainty, defined as the uncertainty of the direct measurement and the indirect determination added in quadrature. Note that in the case of $\alpha_{s}\left(M_{Z}^{2}\right)$ the direct measurement displayed is the world average value [45], which is otherwise not used in the fit.

The availability of the two-loop corrections to the $Z$ partial widths and $\sigma_{\text {had }}^{0}$ allows the determination of $\alpha_{s}\left(M_{Z}^{2}\right)$ to full NNLO and partial NNNLO level. We find

$$
\begin{aligned}
\alpha_{S}\left(M_{Z}^{2}\right)= & 0.1196 \pm 0.0028_{\exp } \pm 0.0006_{\delta_{\text {theo }}} \mathcal{R}_{V, A} \\
& \pm 0.0006_{\delta_{\text {theo }} \Gamma_{i}} \pm 0.0002_{\delta_{\text {theo }} \sigma_{\text {had }}^{0}}^{0} \\
= & 0.1196 \pm 0.0030_{\text {tot }}
\end{aligned}
$$




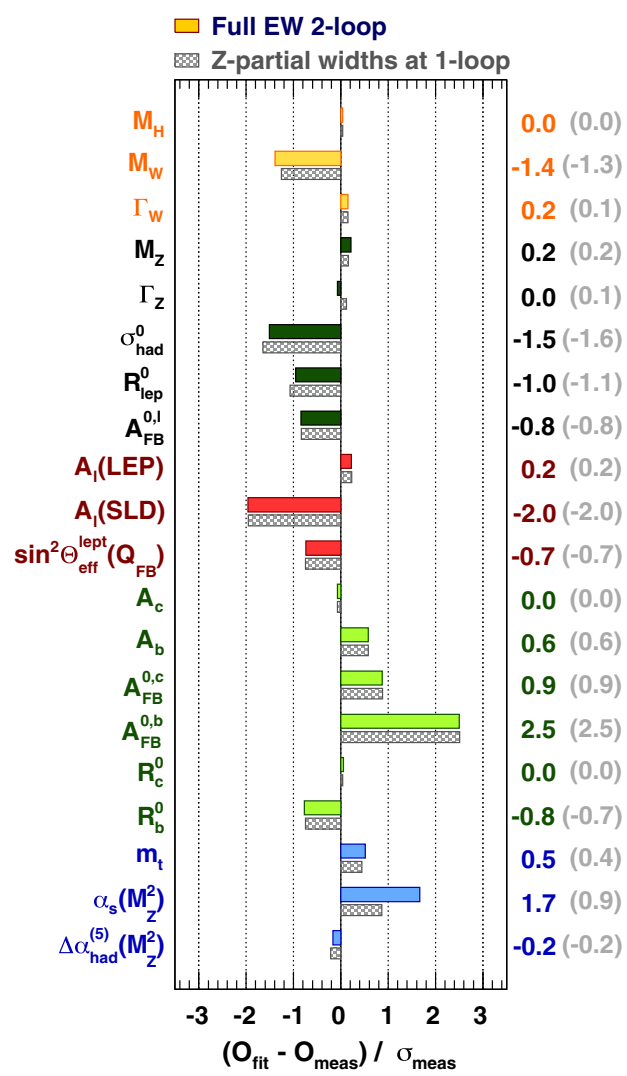

Fig. 1 Left Comparison of the fit results with the direct measurements in units of the experimental uncertainty. The fit results are compared between the scenario using the two-loop calculations of the $Z$ partial widths with the four-loop $\mathcal{O}\left(\alpha_{t} \alpha_{s}^{3}\right)$ correction to $M_{W}$ (colour, top bars) and the one-loop calculation used in a previous publication [4] (shaded grey, bottom bars). Right Comparison of the fit results with the indirect

where the theoretical uncertainties due to missing higherorder contributions are significantly larger than previously estimated [8]. This is largely due to the variation of the full $\mathcal{O}\left(\alpha_{s}^{4}\right)$ terms in the radiator functions and to the uncertainties on the $Z$ partial widths and $\sigma_{\text {had }}^{0}$, not assigned before. The fit indirectly determines the $W$ mass to be

$$
\begin{aligned}
M_{W}= & 80.3584 \pm 0.0046_{m_{t}} \pm 0.0030_{\delta_{\text {theo }} m_{t}} \pm 0.0026_{M_{Z}} \\
& \pm 0.0018_{\Delta \alpha_{\text {had }}} \pm 0.0020_{\alpha_{S}} \pm 0.0001_{M_{H}} \\
& \pm 0.0040_{\delta_{\text {theo }} M_{W}} \mathrm{GeV} \\
= & 80.358 \pm 0.008_{\text {tot }} \mathrm{GeV}
\end{aligned}
$$

providing a result which exceeds the precision of the direct measurement. The different uncertainty contributions originate from the uncertainties on the input values of the fit, as quoted in the second column in Table 2. Simple error propagation is applied to evaluate their impact on the prediction of $M_{W}$. At present, the largest uncertainties are due to $m_{t}$, both experimental and theoretical, followed by the theory and $M_{Z}$ uncertainties.

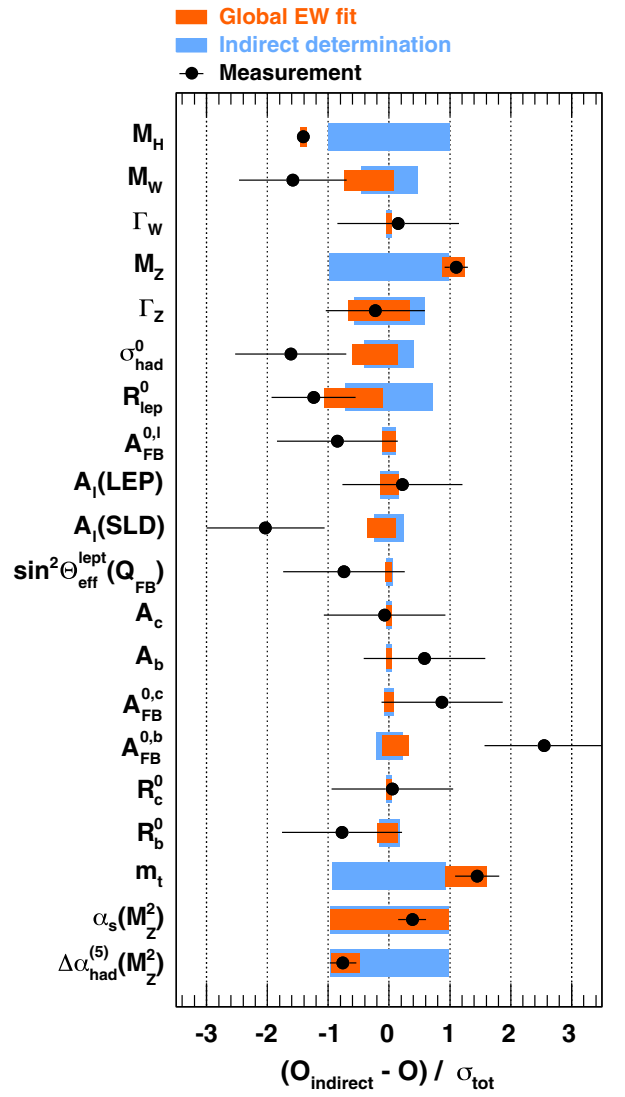

determination in units of the total uncertainty, defined as the uncertainty of the direct measurement and that of the indirect determination added in quadrature. The indirect determination of an observable corresponds to a fit without using the corresponding direct constraint from the measurement

Likewise, the indirect determination of the effective leptonic weak mixing angle, $\sin ^{2} \theta_{\mathrm{eff}}^{\ell}$, gives

$$
\begin{aligned}
\sin ^{2} \theta_{\text {eff }}^{\ell}= & 0.231488 \pm 0.000024_{m_{t}} \pm 0.000016_{\delta_{\text {theo }} m_{t}} \\
& \pm 0.000015_{M_{Z}} \pm 0.000035_{\Delta \alpha_{\text {had }}} \\
& \pm 0.000010_{\alpha_{S}} \pm 0.000001_{M_{H}} \\
& \pm 0.000047_{\delta_{\text {theo }} \sin ^{2} \theta_{\text {eff }}^{f}}, \\
= & 0.23149 \pm 0.00007_{\text {tot }},
\end{aligned}
$$

where the largest uncertainty is theoretical followed by the uncertainties on $\Delta \alpha_{\text {had }}^{(5)}\left(M_{Z}^{2}\right)$ and $m_{t}$.

An important consistency test of the SM is the simultaneous indirect determination of $m_{t}$ and $M_{W}$. A scan of the confidence level (CL) profile of $M_{W}$ versus $m_{t}$ is shown in Fig. 2 (top) for the scenarios where the direct $M_{H}$ measurement is included in the fit (blue) or not (grey). Both contours agree with the direct measurements (green bands and ellipse for two degrees of freedom). The bottom panel of Fig. 2 displays the corresponding CL profile for the observable pair 

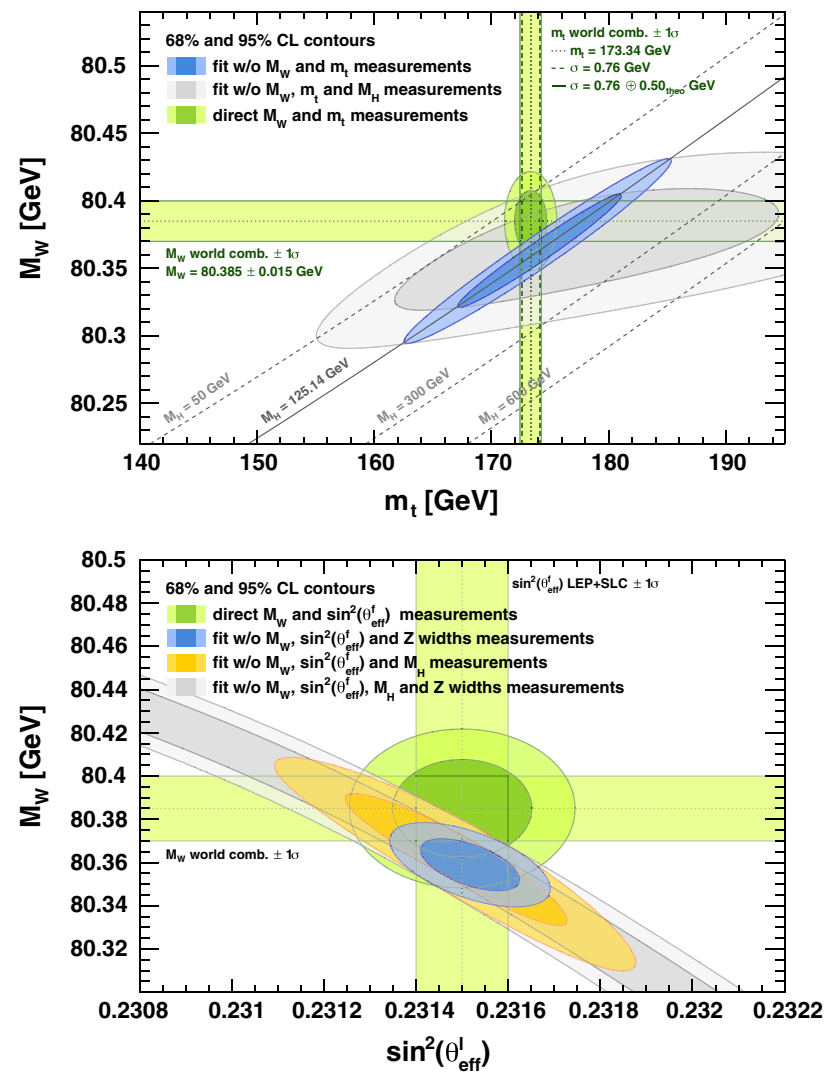

Fig. 2 Contours at 68 and $95 \% \mathrm{CL}$ obtained from scans of $M_{W}$ versus $m_{t}$ (top) and $M_{W}$ versus $\sin ^{2} \theta_{\mathrm{eff}}^{\ell}$ (bottom), for the fit including $M_{H}$ (blue) and excluding $M_{H}$ (grey), as compared to the direct measurements (vertical and horizontal green bands and ellipses). The theoretical uncertainty of $0.5 \mathrm{GeV}$ is added to the direct top-mass measurement. In both figures, the corresponding direct measurements are excluded from the fit. In the case of $\sin ^{2} \theta_{\mathrm{eff}}^{\ell}$, all partial and full $Z$ width measurements are excluded as well (except in case of the orange prediction), besides the asymmetry measurements

$\sin ^{2} \theta_{\text {eff }}^{\ell}$ and $M_{W}$. The coloured ellipses indicate: green for the direct measurements; grey for the electroweak fit without using $M_{W}, \sin ^{2} \theta_{\text {eff }}^{f}, M_{H}$ and the $Z$ width measurements; orange for the fit without using $M_{W}, \sin ^{2} \theta_{\text {eff }}^{f}$ and $M_{H}$; blue for the fit without $M_{W}, \sin ^{2} \theta_{\text {eff }}^{f}$ and the $Z$ width measurements. For both figures the observed agreement demonstrates the consistency of the SM.

Figure 3 shows CL profiles for the observable pair $\sin ^{2} \theta_{\mathrm{eff}}^{\ell}$ and $M_{W}$, but with the theoretical uncertainty on the top mass varied between 0 and $1.5 \mathrm{GeV}$, in steps of $0.5 \mathrm{GeV}$. Assuming a value of $\delta_{\text {theo }} m_{t}=1.5 \mathrm{GeV}$, the uncertainty becomes dominant. It underlines that a better assessment of the theoretical $m_{t}$ uncertainty is of relevance for the fit.

\subsection{Oblique parameters}

If the new physics scale is significantly higher than the electroweak scale, new physics effects from virtual particles in

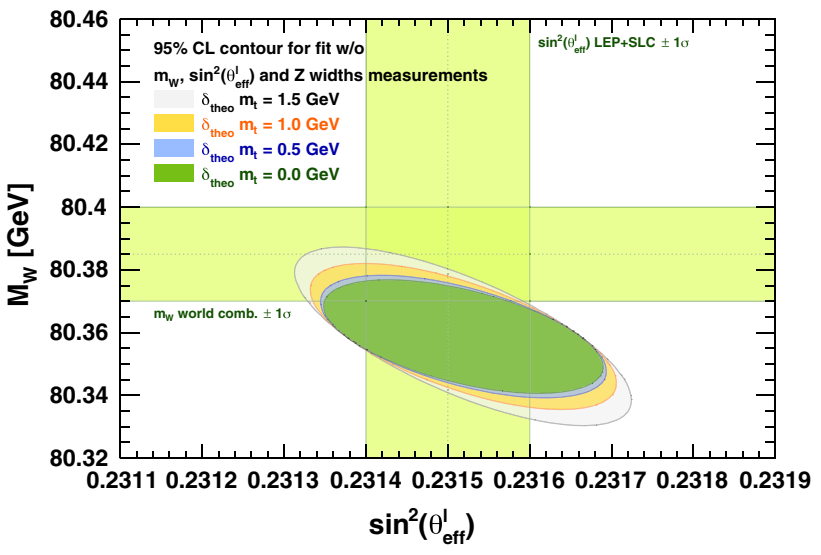

Fig. 3 Contours at $95 \% \mathrm{CL}$ obtained from scans of $M_{W}$ versus $\sin ^{2} \theta_{\text {eff }}^{\ell}$, with the top-mass theoretical uncertainty varied between 0 and $1.5 \mathrm{GeV}$ in steps of $0.5 \mathrm{GeV}$, as compared to the direct measurements (vertical and horizontal green bands). The corresponding direct measurements are excluded from the fit

loops are expected to contribute predominantly through vacuum polarisation corrections to the electroweak precision observables. These terms are traditionally denoted oblique corrections and are conveniently parametrised by the three self-energy parameters $S, T, U[50,51]$. These are defined to vanish in the SM and are closely related to the $\epsilon_{1,2,3}$ parameters [52,53].

The $S$ and $T$ parameters absorb possible new physics contributions to the neutral and to the difference between neutral and charged weak currents, respectively. The $U$ parameter is only sensitive to changes in the mass and width of the $W$ boson. It is very small in most new physics models and therefore often set to zero.

Constraints on the $S, T, U$ parameters can be derived from the global electroweak fit by calculating the difference of the oblique corrections as determined from the experimental data and the corrections obtained from an SM reference point (with fixed reference values of $m_{t}$ and $M_{H}$ ). With this definition significantly non-zero $S, T, U$ parameters represent an unambiguous indication of new physics.

For the studies presented here we use the SM reference as $M_{H, \text { ref }}=125 \mathrm{GeV}$ and $m_{t, \text { ref }}=173 \mathrm{GeV}$. We find

$S=0.05 \pm 0.11, \quad T=0.09 \pm 0.13, \quad U=0.01 \pm 0.11$,

with correlation coefficients of +0.90 between $S$ and $T$, $-0.59(-0.83)$ between $S$ and $U(T$ and $U)$. Fixing $U=0$ one obtains $\left.S\right|_{U=0}=0.06 \pm 0.09$ and $\left.T\right|_{U=0}=0.10 \pm 0.07$, with a correlation coefficient of +0.91 . The constraints on $S$ and $T$ for a fixed value of $U=0$ are shown in Fig. 4. The propagation of the current experimental uncertainties in $M_{H}$ and $m_{t}$ upon the SM prediction is illustrated by the small black area at about $S=T=0$. 


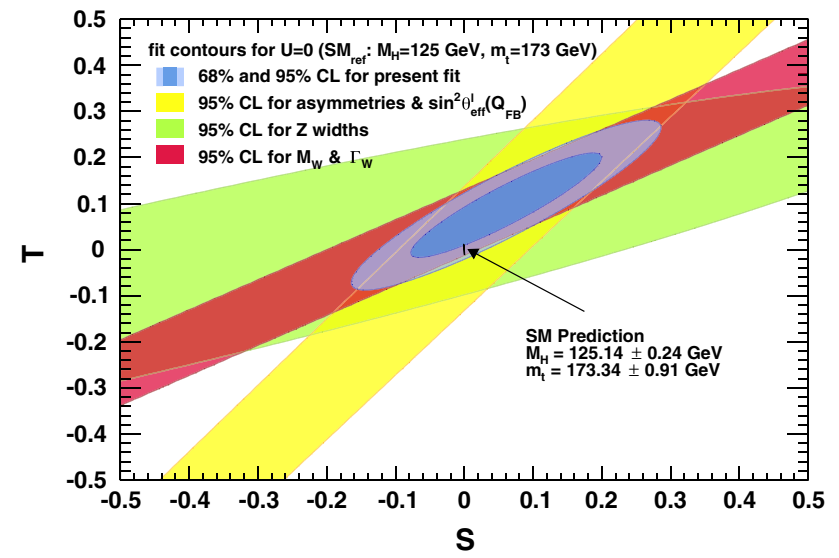

Fig. 4 Constraints on the oblique parameters $S$ and $T$, with the $U$ parameter fixed to zero, using all observables (blue). Individual constraints are shown from the asymmetry measurements (yellow), the $Z$ partial and total widths (green) and $W$ mass and width (red), with confidence levels drawn for one degree of freedom. The SM prediction within uncertainties is indicated by the thin black stroke

\section{Prospects of the electroweak fit with the LHC and ILC/GigaZ}

We use a simplified set of input observables to study the prospects of the electroweak fit for the Phase-1 LHC and the ILC/GigaZ. The measurements of the $Z$ pole asymmetry observables are summarised in a single value of the effective weak mixing angle. The measurement of $R_{\ell}^{0}$ is the only partial decay width that enters the fit to constrain $\alpha_{S}$. This simplified fit setup leads in some cases to reduced constraints on observables as can be seen by comparing the uncertainties of the present scenarios between the last column of Table 2 and the fifth column of Table 3 . The central values of the observables are adjusted to the values predicted by the current best fit giving a fully consistent set of SM observables. ${ }^{6}$

\subsection{Experimental and theoretical improvements}

For the LHC, with a large dataset and sufficient time to understand and improve systematic uncertainties, we assume the following scenario.

- For $m_{H}$ an uncertainty of $100 \mathrm{MeV}$ is assumed, although the experiments are expected to exceed this precision using, for example, Higgs decays to four muons. Whatever uncertainty used is irrelevant for the fits discussed.

- A precision of $10 \mathrm{MeV}$ on $M_{W}$ may be achievable for the final combination of Tevatron measurements [54].

\footnotetext{
6 The following central values are used for the future scenarios: $M_{H}=$ $125.0 \mathrm{GeV}, \Delta \alpha_{\text {had }}^{(5)}\left(M_{Z}^{2}\right)=2755.4 \times 10^{-5}, M_{Z}=91.1879 \mathrm{GeV}, m_{t}=$ $173.81 \mathrm{GeV}, M_{W}=80.363 \mathrm{GeV}, \sin ^{2} \theta_{\mathrm{eff}}^{\ell}=0.231492$ and $R_{\ell}^{0}=$ 20.743. See Table 3 for the corresponding uncertainties.
}

Assuming improvements in the uncertainties due to parton distribution functions, the modelling of the lepton transverse momentum and a reduction of experimental uncertainties, we expect that a combined precision of $8 \mathrm{MeV}$ may be in reach for a combination of the LHC, Tevatron and LEP results.

- Given the present combined $m_{t}$ uncertainty of $0.76 \mathrm{GeV}$ [47], we assume an ultimate experimental precision of $0.6 \mathrm{GeV}$ as a long-term prospect. As discussed earlier, an additional theoretical uncertainty of $0.5 \mathrm{GeV}$ is assigned. For the future LHC scenario, with further theoretical studies on the top-mass ambiguity, additional high-statistics tests of top-quark decay kinematics and a possible perturbative four-loop relation between pole and $\overline{\mathrm{MS}}$ mass $[41,44]$, this uncertainty is assumed to be reduced to $0.25 \mathrm{GeV}$.

For the ILC/GigaZ we assume the following benchmark uncertainties. $^{7}$

- A precision of $5 \mathrm{MeV}$ is assumed for $M_{W}$, obtained from cross section measurements at and above the $W W$ production threshold [11].

- Scans of the $t \bar{t}$ production threshold are expected to yield an experimental precision on the top-quark mass of approximately $30 \mathrm{MeV}[11,44]$. The conversion of the threshold to an $\overline{\mathrm{MS}}$ mass using perturbative QCD adds an estimated uncertainty of $100 \mathrm{MeV}[11,40,44]$, which is used as theoretical uncertainty $\delta_{\text {theo }} m_{t}$ in this scenario.

- Measurements of the weak left-right asymmetry $A_{\mathrm{LR}}$ from leptonic and hadronic $Z$ decays are expected to yield a precision of $1.3 \times 10^{-5}$ for $\sin ^{2} \theta_{\text {eff }}^{f}$ [11], improving the present measurement combination by more than a factor of 10 .

- The partial decay width of the $Z$ boson, $R_{\ell}^{0}$, is assumed to be measured with a precision of $4 \times 10^{-3}$, improving the current measurement [55] by a factor of more than 6 .

For both future scenarios we assume that the uncertainty in $\Delta \alpha_{\text {had }}^{(5)}\left(M_{Z}^{2}\right)$ will reduce from currently $10 \times 10^{-5}$ down to $4.7 \times 10^{-5}$. The improvement is expected due to updated $e^{+} e^{-} \rightarrow$ hadrons cross section measurements below the charm threshold from the completion of ongoing BABAR and VEPP-2000 analyses, improved charmonium resonance data from BES-III and a better knowledge of $\alpha_{S}$ from reliable Lattice QCD predictions [56].

\footnotetext{
7 An improvement in the $M_{Z}$ precision from currently 2.1 to $1.6 \mathrm{MeV}$ is suggested in [11]. Such a measurement would require the knowledge of the absolute ILC beam energy with a precision of $10^{-5}$. Since the technical feasibility of such a precision is still uncertain, we do not yet include it in the fit.
} 
Table 3 Current and extrapolated future uncertainties in the input observables (left) and the precision obtained for the fit prediction (right). Where two uncertainties are given, the first is experimental and the second theoretical. The value of $\alpha_{S}\left(M_{Z}^{2}\right)$ is not used directly as input in the fit. The uncertainty in the direct $M_{H}$ measurements is not relevant for the fit and therefore not quoted. For all indirect determinations shown (including the present $M_{H}$ determination) the assumed central values of the input measurements have been adjusted to obtain a common fit value of $M_{H}=125 \mathrm{GeV}$. The simplified fit setup used to derive the numbers in this table leads in some cases to reduced constraints on observables as can be seen by comparing the uncertainties of the present scenarios (fifth column) with the last column of Table 2. See text for more details

\begin{tabular}{|c|c|c|c|c|c|c|}
\hline \multirow[t]{2}{*}{ Parameter } & \multicolumn{3}{|c|}{ Experimental input $\left[ \pm 1 \sigma_{\exp }\right]$} & \multicolumn{3}{|c|}{ Indirect determination $\left[ \pm 1 \sigma_{\text {exp }}, \pm 1 \sigma_{\text {theo }}\right]$} \\
\hline & Present & LHC & ILC/GigaZ & Present & LHC & ILC/GigaZ \\
\hline$M_{H}[\mathrm{GeV}]$ & 0.2 & $<0.1$ & $<0.1$ & $\begin{array}{l}+31,+10 \\
-26,-8\end{array}$ & $\begin{array}{l}+20,+3.9 \\
-18,-3.2\end{array}$ & $\begin{array}{ll}+6.8 & +2.5 \\
-6.5 & -2.4\end{array}$ \\
\hline$M_{W}[\mathrm{MeV}]$ & 15 & 8 & 5 & $6.0,5.0$ & $5.2,1.8$ & $1.9,1.3$ \\
\hline$M_{Z}[\mathrm{MeV}]$ & 2.1 & 2.1 & 2.1 & 11,4 & $7.0,1.4$ & $2.5,1.0$ \\
\hline$m_{t}[\mathrm{GeV}]$ & 0.8 & 0.6 & 0.1 & $2.4,0.6$ & $1.5,0.2$ & $0.7,0.2$ \\
\hline $\sin ^{2} \theta_{\mathrm{eff}}^{\ell}\left[10^{-5}\right]$ & 16 & 16 & 1.3 & $4.5,4.9$ & $2.8,1.1$ & $2.0,1.0$ \\
\hline$\Delta \alpha_{\text {had }}^{5}\left(M_{Z}^{2}\right)\left[10^{-5}\right]$ & 10 & 4.7 & 4.7 & 42,13 & 36,6 & $5.6,3.0$ \\
\hline$R_{l}^{0}\left[10^{-3}\right]$ & 25 & 25 & 4 & - & - & - \\
\hline$\alpha_{S}\left(M_{Z}^{2}\right)\left[10^{-4}\right]$ & - & - & - & 40,10 & 39,7 & $6.4,6.9$ \\
\hline$\left.S\right|_{U=0}$ & - & - & - & $0.094,0.027$ & $0.086,0.006$ & $0.017,0.006$ \\
\hline$\left.T\right|_{U=0}$ & - & - & - & $0.083,0.023$ & $0.064,0.005$ & $0.022,0.005$ \\
\hline$\kappa_{V}(\lambda=3 \mathrm{TeV})$ & 0.05 & 0.03 & 0.01 & 0.02 & 0.02 & 0.01 \\
\hline
\end{tabular}

The present and projected experimental uncertainties for the observables used in the simplified electroweak fit are summarised in the left columns of Table 3.

To match the experimental precision significant theoretical progress is required. Leaving aside the ambiguity in $m_{t}$ discussed above, the presently most important theoretical uncertainties affecting the fit are those related to the predictions of $M_{W}$ and $\sin ^{2} \theta_{\text {eff }}^{f}$. For the future scenarios, we assume that the present uncertainties of $\delta_{\text {theo }} M_{W}=4 \mathrm{MeV}$ and $\delta_{\text {theo }} \sin ^{2} \theta_{\text {eff }}^{f}=4.7 \times 10^{-5}$ reduce to $1 \mathrm{MeV}$ and $10^{-5}$, respectively. This reduction will require ambitious three-loop electroweak calculations. The leading theoretical uncertainties on the partial $Z$ decay widths, $\sigma_{\text {had }}^{0}$, and the radiator functions play a smaller role in the present fit. For the future scenarios the uncertainty estimates given in Table 1 are assumed to be reduced by a factor of 4 , similar to the uncertainties on $M_{W}$ and $\sin ^{2} \theta_{\text {eff }}^{f}$.

\subsection{Expected fit performance}

The numerical $1 \sigma$ uncertainties of the indirect observable determinations are given for the present fit as well as the LHC and ILC/GigaZ scenarios in the right-hand columns of Table 3. Experimental and theoretical uncertainties are quoted separately.

Examples of $\Delta \chi^{2}$ profiles for three key observables are shown in Fig. 5. Throughout this section, blue, green and orange curves indicate the present, future LHC and future ILC/GigaZ scenarios. The impact of the theoretical uncertainties is illustrated by the width of each coloured curve.
The light blue curve in the top panel in Fig. 5 indicates the $M_{H}$ constraint using the present precision, but with the central experimental values adjusted to the future scenarios. It allows a direct comparison with the present uncertainties, which depend on the value of $M_{H}$.

If the extrapolated precision on $M_{W}$ and $m_{t}$ can be realised, the LHC will significantly improve the indirect constraint on $M_{H}$ (present at $M_{H} \simeq 125 \mathrm{GeV}:{ }_{-27}^{+33} \mathrm{GeV}$, LHC: $\left.{ }_{-18}^{+21} \mathrm{GeV}\right)$. An even more substantial improvements is expected for the ILC/GigaZ with an expected uncertainty of ${ }_{-7.0}^{+7.4} \mathrm{GeV}^{8}$

Correspondingly, the prediction of $M_{W}$ from the fit (see middle panel of Fig. 5) can be improved by the LHC (reduced uncertainty from currently 7.8 to $5.5 \mathrm{MeV}$, owing also to the reduced theoretical uncertainties) and by the ILC/GigaZ $(2.3 \mathrm{MeV})$. Also shown on the figure are the current and expected future direct measurements, keeping the central value unchanged. A powerful SM test is obtained, confronting measurement and prediction of $M_{W}$ at the level of 0.05 per mill.

The prediction of $\sin ^{2} \theta_{\text {eff }}^{\ell}$ from the fit (bottom panel of Fig. 5) is significantly improved in the LHC and ILC/GigaZ scenarios, also owing to the improved theoretical precision. The total uncertainty reduces from currently $6.6 \times 10^{-5}$ by

\footnotetext{
${ }^{8}$ If the experimental input data, currently predicting $M_{H}=$ $94_{-22}^{+25} \mathrm{GeV}$, are left unchanged with respect to the present central values but had uncertainties according to the future expectations, a precision of ${ }_{-14}^{+16} \mathrm{GeV}$ and ${ }_{-5.3}^{+5.6} \mathrm{GeV}$ is obtained for LHC and ILC/GigaZ, respectively. A deviation of the measured $M_{H}$ at a level of $5 \sigma$ could be established with the ILC/GigaZ fit.
} 

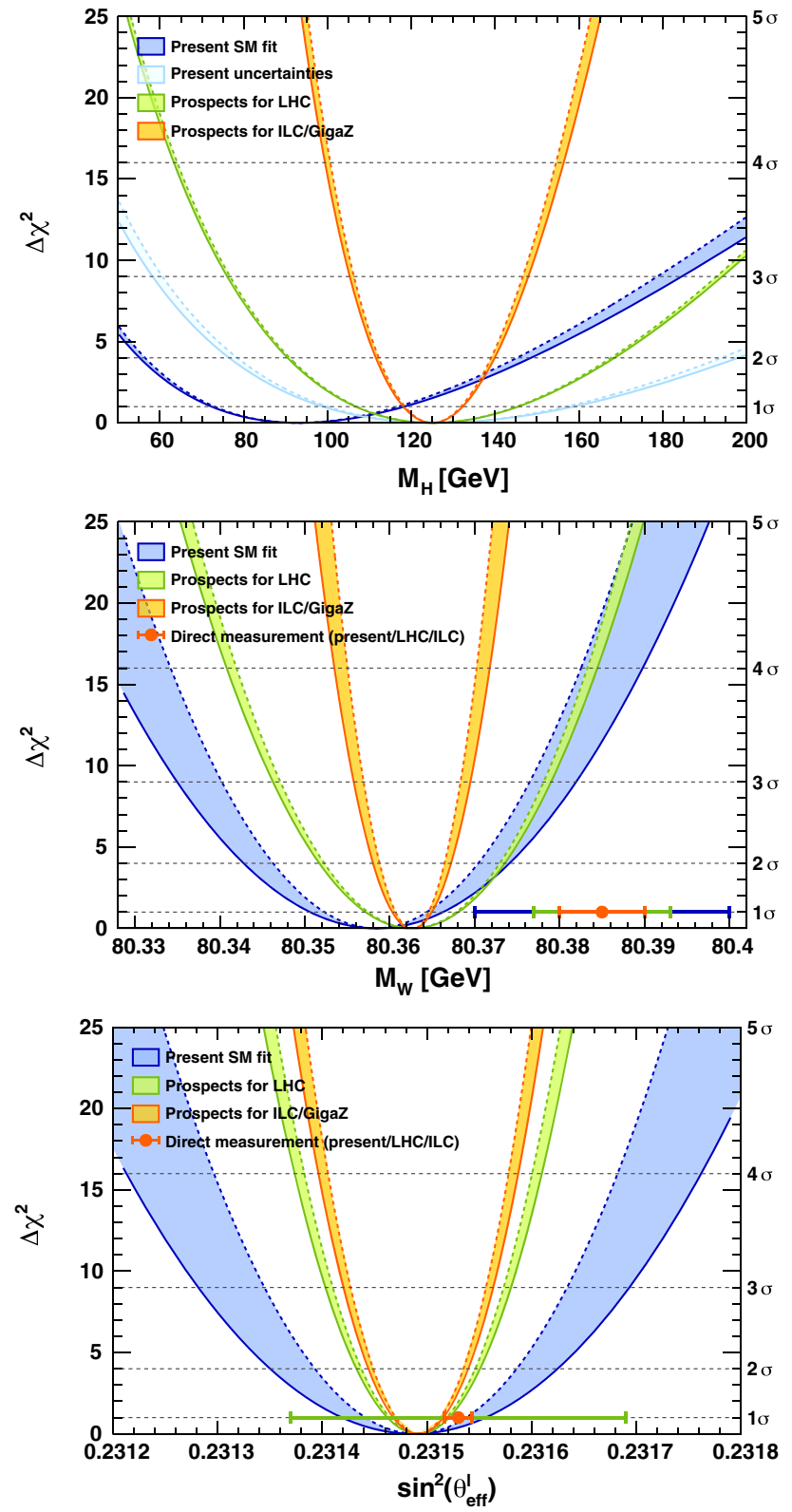

Fig. 5 Profiles of $\Delta \chi^{2}$ versus $M_{H}$ (top), $M_{W}$ (middle) and $\sin ^{2} \theta_{\mathrm{eff}}^{\ell}$ (bottom). In blue the present result and in light blue, green and orange the present, LHC and ILC/GigaZ scenarios, respectively, all using the future fit setup (reproducing $M_{H} \simeq 125 \mathrm{GeV}$ ) with corresponding uncertainties. The impact of the theoretical uncertainties is illustrated by the width of the coloured curves. See Table 3 for the numerical results of these fits

almost a factor of 3 at the ILC/GigaZ. Again the current and expected future direct measurements are also indicated on the figure, keeping the central value unchanged. No improvement in the precision of the direct measurement is expected from the LHC, leaving the direct measurement a factor 5 less precise than the indirect determination. Only within the ILC/GigaZ scenario a similar precision between the prediction and direct measurement can be achieved.
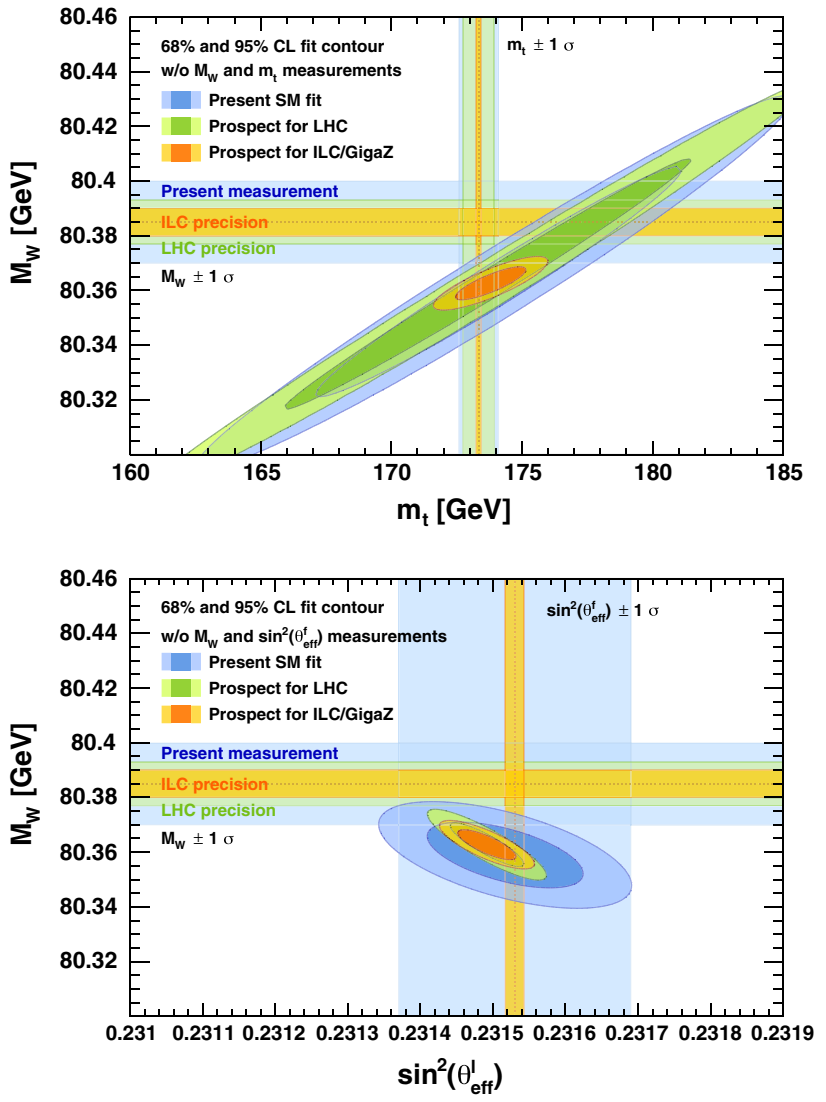

Fig. 6 Fit constraints for the present and extrapolated future scenarios compared to the direct measurements for the observable pairs $M_{W}$ versus $m_{t}$ (top) and $M_{W}$ versus $\sin ^{2} \theta_{\text {eff }}^{\ell}$ (bottom). The direct measurements are not included as input measurements in the fits. For the future scenarios the central values of the other input measurements are adjusted to reproduce the SM with $M_{H} \simeq 125 \mathrm{GeV}$. The horizontal and vertical bands indicate in blue today's precision of the direct measurements and in light green and orange the extrapolated precisions for the LHC and ILC/GigaZ, respectively. The ellipses receive significant contributions from the theoretical uncertainties parametrised by $\delta_{\text {theo }} M_{W}$ and $\delta_{\text {theo }} \sin ^{2} \theta_{\text {eff }}^{f}$. For better visibility the measurement ellipses corresponding to two degrees of freedom are not drawn

Figure 6 shows the allowed areas obtained for fits with fixed variable pairs $M_{W}$ versus $m_{t}$ (top) and $M_{W}$ versus $\sin ^{2} \theta_{\text {eff }}^{\ell}$ (bottom) in the three scenarios. The horizontal and vertical bands display the $1 \sigma$ ranges of the current direct measurements (blue), as well as the LHC (green) and ILC/GigaZ (orange) expectations in precision. A modest improvement in precision is achieved for the LHC, represented by the green ellipses, when confronting the direct measurements with the SM predictions. A much stronger increase in precision and sensitivity is obtained with the ILC/GigaZ (orange ellipses).

\subsection{Impact of the individual uncertainties}

Table 4 shows a breakdown of the predicted uncertainties of various parameters as obtained from the reduced elec- 
Table 4 Contributions from the individual experimental and theoretical uncertainty sources to the total uncertainty in the indirect determination of a given observable by the electroweak fit for the three scenarios (present, future LHC, ILC/GigaZ). The uncertainty due to $M_{H}$ is negligible compared to the other observables and is not shown. See text for further discussion

\begin{tabular}{|c|c|c|c|c|c|c|c|c|c|c|}
\hline \multirow[t]{2}{*}{ Parameter } & \multirow[t]{2}{*}{$\delta_{\text {meas }}$} & \multirow[t]{2}{*}{$\delta_{\text {fit }}^{\text {tot }}$} & \multirow[t]{2}{*}{$\delta_{\text {fit }}^{\text {theo }}$} & \multirow[t]{2}{*}{$\delta_{\text {fit }}^{\exp }$} & \multicolumn{6}{|c|}{ Experimental uncertainty source $[ \pm 1 \sigma]$} \\
\hline & & & & & $\delta M_{W}$ & $\delta M_{Z}$ & $\delta m_{t}$ & $\delta \sin ^{2} \theta_{\text {eff }}^{f}$ & $\delta \Delta \alpha_{\text {had }}$ & $\delta \alpha_{S}$ \\
\hline \multicolumn{11}{|c|}{ Present uncertainties } \\
\hline$M_{H}[\mathrm{GeV}]$ & 0.2 & $\begin{array}{l}+33 \\
-27\end{array}$ & ${ }_{-8}^{+10}$ & $\begin{array}{l}+31 \\
-26\end{array}$ & $\begin{array}{l}+28 \\
-23\end{array}$ & $\begin{array}{l}+5 \\
-4\end{array}$ & ${ }_{-7}^{+10}$ & $\begin{array}{l}+29 \\
-23\end{array}$ & $\begin{array}{l}+7 \\
-5\end{array}$ & $\begin{array}{l}+4 \\
-3\end{array}$ \\
\hline$M_{W}[\mathrm{MeV}]$ & 15 & 7.8 & 5.0 & 6.0 & - & 2.5 & 4.3 & 5.1 & 1.6 & 2.5 \\
\hline$M_{Z}[\mathrm{MeV}]$ & 2.1 & 12.0 & 3.7 & 11.4 & 10.5 & - & 3.5 & 11.2 & 2.2 & 1.4 \\
\hline$m_{t}[\mathrm{GeV}]$ & 0.8 & 2.5 & 0.6 & 2.4 & 2.3 & 0.4 & - & 2.3 & 0.5 & 0.6 \\
\hline $\sin ^{2} \theta_{\mathrm{eff}}^{\ell}$ a & 16 & 6.6 & 4.9 & 4.5 & 3.7 & 1.2 & 2.0 & - & 3.4 & 1.2 \\
\hline$\Delta \alpha_{\text {had }}{ }^{\text {a }}$ & 10 & 44 & 13 & 42 & 31 & 6 & 10 & 41 & - & 2 \\
\hline \multicolumn{11}{|c|}{ LHC prospects } \\
\hline$M_{H}[\mathrm{GeV}]$ & $<0.1$ & $\begin{array}{l}+21 \\
-18\end{array}$ & $\begin{array}{l}+4 \\
-3\end{array}$ & $\begin{array}{l}+20 \\
-18\end{array}$ & $\begin{array}{l}+17 \\
-14\end{array}$ & $\begin{array}{l}+6 \\
-5\end{array}$ & $\begin{array}{l}+8 \\
-7\end{array}$ & $\begin{array}{l}+18 \\
-16\end{array}$ & $\begin{array}{l}+3 \\
-2\end{array}$ & $\begin{array}{l}+5 \\
-4\end{array}$ \\
\hline$M_{W}[\mathrm{MeV}]$ & 8 & 5.5 & 1.8 & 5.2 & - & 2.5 & 3.5 & 4.8 & 0.8 & 2.6 \\
\hline$M_{Z}[\mathrm{MeV}]$ & 2.1 & 7.2 & 1.4 & 7.0 & 6.0 & - & 2.8 & 5.9 & 0.8 & 1.9 \\
\hline$m_{t}[\mathrm{GeV}]$ & 0.6 & 1.5 & 0.2 & 1.5 & 1.3 & 0.4 & - & 1.2 & 0.2 & 0.5 \\
\hline $\sin ^{2} \theta_{\mathrm{eff}}^{\ell}{ }^{\mathrm{a}}$ & 16 & 3.0 & 1.1 & 2.8 & 2.5 & 1.1 & 1.4 & - & 1.5 & 0.9 \\
\hline$\Delta \alpha_{\text {had }}{ }^{\text {a }}$ & 4.7 & 36 & 6 & 36 & 25 & 9 & 12 & 35 & - & 5 \\
\hline \multicolumn{11}{|c|}{ ILC/GigaZ prospects } \\
\hline$M_{H}[\mathrm{GeV}]$ & $<0.1$ & $\begin{array}{l}+7.3 \\
-6.9\end{array}$ & $\begin{array}{l}+2.5 \\
-2.4\end{array}$ & $\begin{array}{l}+6.8 \\
-6.5\end{array}$ & $\begin{array}{l}+2.5 \\
-3.6\end{array}$ & $\begin{array}{l}+4.3 \\
-4.0\end{array}$ & $\begin{array}{l}+0.3 \\
-0.2\end{array}$ & $\begin{array}{l}+3.4 \\
-2.9\end{array}$ & $\begin{array}{l}+4.3 \\
{ }_{-4.0}\end{array}$ & $\begin{array}{l}+0.3 \\
-0.3\end{array}$ \\
\hline$M_{W}[\mathrm{MeV}]$ & 5 & 2.3 & 1.3 & 1.9 & - & 1.7 & 0.1 & 1.2 & 0.6 & 0.3 \\
\hline$M_{Z}[\mathrm{MeV}]$ & 2.1 & 2.7 & 1.0 & 2.5 & 2.4 & - & 0.1 & 1.3 & 1.9 & 0.2 \\
\hline$m_{t}[\mathrm{GeV}]$ & 0.1 & 0.8 & 0.2 & 0.7 & 0.6 & 0.5 & - & 0.3 & 0.4 & 0.2 \\
\hline $\sin ^{2} \theta_{\text {eff }}^{\ell}{ }^{a}$ & 1.3 & 2.3 & 1.0 & 2.0 & 1.7 & 1.2 & 0.1 & - & 1.5 & 0.1 \\
\hline$\Delta \alpha_{\text {had }}{ }^{\text {a }}$ & 4.7 & 6.4 & 3.0 & 5.6 & 2.6 & 4.2 & 0.2 & 3.8 & - & 0.2 \\
\hline
\end{tabular}

${ }^{\text {a }}$ In units of $10^{-5}$

troweak fit for the present and future scenarios. The present and prospective experimental precision of the direct measurement, $\delta_{\text {meas }}$, are given in column two. In column three the total uncertainty from the indirect determination, i.e. the result from a fit without using the experimental observable of that row, $\delta_{\text {fit }}^{\text {tot }}$, is given. The contributions from the theoretical uncertainties, $\delta_{\text {fit }}^{\text {theo }}$, and experimental uncertainties, $\delta_{\text {fit }}^{\exp }$, are shown in columns four and five. Columns 6 to 11 give the uncertainties of the indirect fit determination resulting from the experimental uncertainties of the observables listed in the respective columns. These uncertainties are obtained as the difference between the result obtained from the full fit and the result when excluding the experimental uncertainty given in that column. In this approach the correlations between the fit parameters are neglected, such that the individual experimental uncertainties do not add up in quadrature to the full experimental uncertainty as obtained from the fit. The given individual uncertainties thus show the precision that can be gained by improving the constraints from a single measurement.
One notices that the dominant uncertainty contributions vary between the three scenarios. For $M_{H}$, the precision on the indirect determination is presently dominated by the uncertainty on the measurements of $\sin ^{2} \theta_{\text {eff }}^{f}$ and $M_{W}$, which does not change for the LHC scenario. For the ILC/GigaZ, however, the uncertainties on $M_{Z}$ and $\Delta \alpha_{\text {had }}^{(5)}\left(M_{Z}^{2}\right)$ become equally important and a total precision of less than $10 \mathrm{GeV}$ can be achieved. For $M_{W}$, improvements in the theoretical uncertainty and on $\delta m_{t}$ could lead to a precision of $5.5 \mathrm{MeV}$ for the LHC scenario and of $2.3 \mathrm{MeV}$ for the ILC/GigaZ. This would exceed the present experimental precision by 60 to $75 \%$, respectively. For $\sin ^{2} \theta_{\text {eff }}^{\ell}$, improvements in the theoretical uncertainty and in $\Delta \alpha_{\text {had }}^{(5)}\left(M_{Z}^{2}\right)$ and $m_{t}$ are expected, and they could lead to a precision on $\sin ^{2} \theta_{\mathrm{eff}}^{\ell}$ of $3.0 \times 10^{-5}$ for the future LHC scenario, which would exceed the present experimental precision by more than a factor of 5. The ILC/GigaZ would rectify the imbalance in precision: a precision of $1.3 \times 10^{-5}$ for the direct measurement would confront an indirect determination with $2.3 \times 10^{-5}$ total uncertainty. 


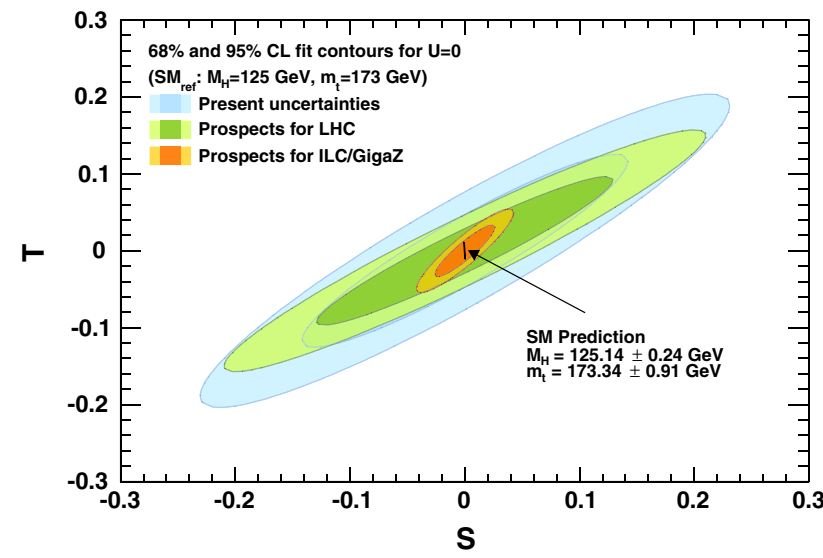

Fig. 7 Comparison of the present (light blue), the LHC (green) and the ILC/GigaZ prospects (orange) on the oblique parameters $S$ and $T$, with the $U$ parameter fixed to zero. The shift in the position of the ellipses between the present data and future scenarios is caused by the different central values used for the electroweak observables in these scenarios. The future scenarios are by construction centred at $S=T=0$. The SM prediction within uncertainties is indicated by the thin black stroke

At the ILC/GigaZ a comparable precision between direct determination and fit constraint would be reached for $M_{Z}$ and $\Delta \alpha_{\text {had }}^{(5)}\left(M_{Z}^{2}\right)$, owing to the improved precision on $M_{W}$ and $\sin ^{2} \theta_{\mathrm{eff}}^{f}$. Also, an indirect constraint on $m_{t}$ of $1 \mathrm{GeV}$ would be possible.

Independently from the other improvements, the determination of $\alpha_{S}\left(M_{Z}^{2}\right)$ from $R_{\ell}^{0}$ would also greatly benefit from the ILC/GigaZ. The current $\alpha_{S}\left(M_{Z}^{2}\right)$ precision of $30 \times 10^{-4}$ is dominated by experimental uncertainties and could be improved to $9 \times 10^{-4}$. It would be the most precise experimental determination of the strong coupling constant, only challenged by calculations from Lattice QCD.

\subsection{Prospects for the oblique parameter determination}

The expected future constraints on $S$ and $T$ for a fixed value of $U=0$ are shown in Fig. 7. The results from the fit of the present scenario with central values adjusted to obtain $M_{H} \simeq 125 \mathrm{GeV}$ are shown in light blue. The shift in the central values between the light blue ellipse and the results shown in Fig. 4 originate from the different central values used for the electroweak observables. By construction the ellipses are centred around $S=T=0$. The uncertainties in the present scenario are larger by about 0.01 in $S$ and 0.02 in $T$ due to the reduced list of observables used in the prospective fit, as discussed in the beginning of this section.

Compared to the present scenario only a minor improvement is expected for the LHC scenario. A reduction of the uncertainty by a factor of 3 to 4 is, however, expected for the ILC/GigaZ. The numerical values of the uncertainties on $S$ and $T$ are given in Table 3. The parameters $S$ and $T$ are strongly correlated, with correlation coefficients of $0.93,0.96$ and 0.91 for the present, LHC and ILC/GigaZ scenarios.

Additional variables like the total width of the $Z, \Gamma_{Z}$, which could be measured to an accuracy of $0.8 \mathrm{MeV}$ at the ILC/GigaZ [11], improve the precision on $\delta S$ and $\delta T$ by about $10 \%$.

\section{Status and prospects for the Higgs couplings determination}

To test the validity of the SM and look for signs of new physics, precision measurements of the properties of the Higgs boson are of critical importance. Key are the couplings to the SM fermions and bosons, which are predicted to depend linearly on the fermion mass and quadratically on the boson mass.

Modified Higgs couplings have been probed by ATLAS and CMS in various benchmark models [57-64]. These employ an effective theory approach, where higher-order modifiers to a phenomenological Lagrangian are matched at tree-level to the SM Higgs boson couplings. In one popular model all boson and all fermion couplings are modified in the same way, scaled by the constants $\kappa_{V}$ and $\kappa_{F}$, respectively, where $\kappa_{V}=\kappa_{F}=1$ for the SM. This benchmark model uses the explicit assumption that no other new physics is present, e.g., there are no additional loops in the production or decay of the Higgs boson and no invisible Higgs decays and undetectable contributions to its decay width. For details see Ref. [65].

The combined analysis of electroweak precision data and Higgs signal-strength measurements has been studied by several groups $[5,9,66-71]$. The main effect of this model on the electroweak precision observables is from the modified Higgs coupling to gauge bosons, and manifests itself through loop diagrams involving the longitudinal degrees of freedom of these bosons. The corrections to the $Z$ and $W$ boson propagators can be expressed in terms of the $S, T$ parameters [66],

$$
\begin{aligned}
& S=\frac{1}{12 \pi}\left(1-\kappa_{V}^{2}\right) \ln \frac{\Lambda^{2}}{M_{H}^{2}}, \\
& T=-\frac{3}{16 \pi \cos ^{2} \theta_{\mathrm{eff}}^{\ell}}\left(1-\kappa_{V}^{2}\right) \ln \frac{\Lambda^{2}}{M_{H}^{2}}, \\
& \Lambda=\frac{\lambda}{\sqrt{\left|1-\kappa_{V}^{2}\right|}},
\end{aligned}
$$

and $U=0$. The cut-off scale $\Lambda$ represents the mass scale of the new states that unitarise longitudinal gauge-boson scattering, as required in this model. Note that the less $\kappa_{V}$ deviates from 1, the higher the scale of new physics. Most BSM models with additional Higgs bosons giving positive corrections 
Higgs-vector boson couplings scaling
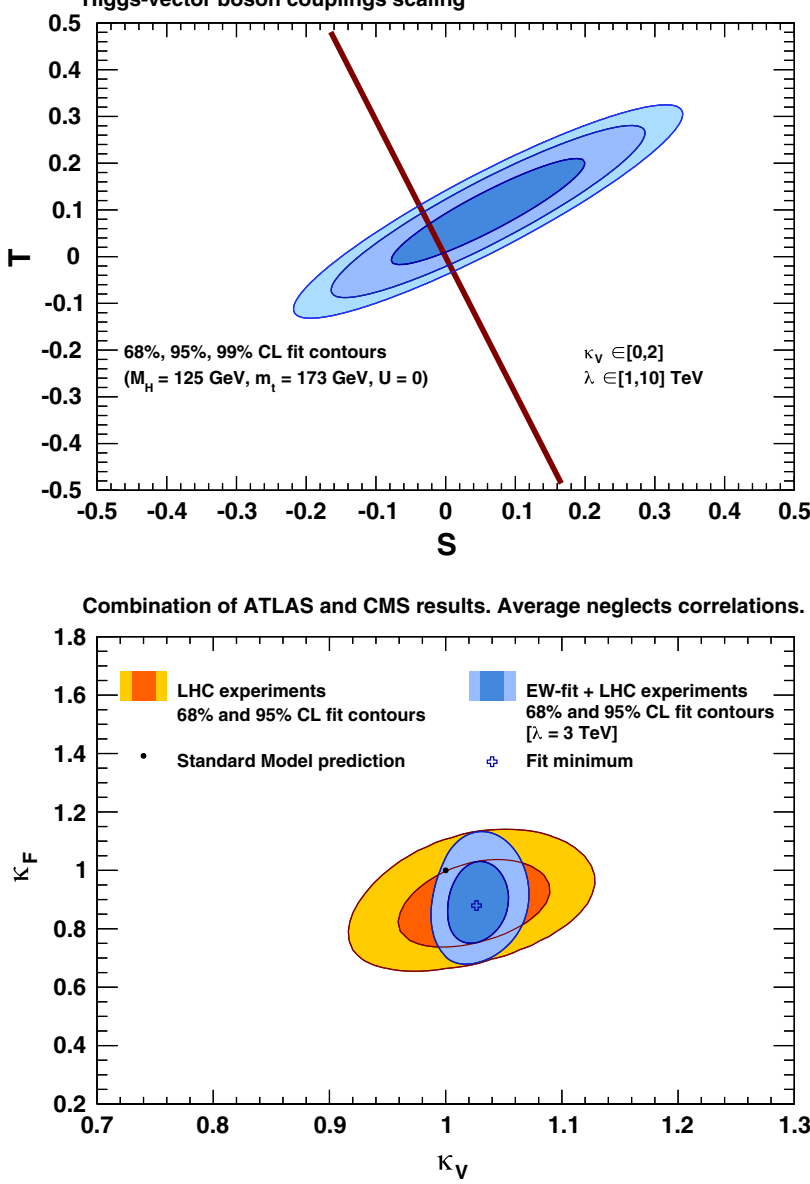

Fig. 8 Top Contour lines of 68 and $95 \%$ CL allowed regions for fixed values of $S$ and $T$ with $U=0$ for the present data (blue). Overlaid (dark red) is the predicted line for $S$ and $T$ for $\kappa_{V} \in[0,2]$ and $\lambda \in[1,10] \mathrm{TeV}$. Bottom: Measurement of $\kappa_{F}$ versus $\kappa_{V}$ at 68 and $95 \% \mathrm{CL}$ from a private combination of present ATLAS and CMS results (orange), overlaid with the constraint of $\kappa_{F}$ versus $\kappa_{V}$ when including the EW-fit (blue)

to the $W$ mass predict values of $\kappa_{V}$ smaller than 1 . Here the nominator $\lambda$ is varied between 1 and $10 \mathrm{TeV}$ and is nominally fixed to $3 \mathrm{TeV}(4 \pi v)$.

Figure 8 (top) shows the predictions for $S$ and $T$, profiled over $\kappa_{V}$ and $\lambda$, together with the allowed regions for $S$ and $T$ from the current electroweak fit. The length of the predicted line covers a variation in $\kappa_{V}$ between $[0,2]$, the width covers the variation in $\lambda$.
The bottom panel of Fig. 8 shows $\kappa_{V}$ and $\kappa_{F}$ as obtained from a private combination of ATLAS and CMS results using all publicly available information on the measured Higgs signal-strength modifiers $\mu_{i}$. Also shown is the combined constraint on $\kappa_{V}$ (and $\kappa_{F}$ ) from the LHC experiments and the electroweak fit.

The published Higgs coupling measurements of $\mu_{\mathrm{ggF}+\mathrm{ttH}}$ versus $\mu_{\mathrm{VBF}+\mathrm{VH}}$ from ATLAS and CMS used in this combination are summarised in Table 5. The measurements from the ATLAS Higgs to di-boson channels are published likelihood scans [57]. The CMS results in Table 5 are approximate values derived from public likelihood iso-contour lines. Correlations of the theory and detector related uncertainties between the various $\mu_{i}$ are neglected in the combination, as these are not provided by the experiments. We find that the individual experimental combinations of ATLAS and CMS for $\kappa_{V}$ (and $\kappa_{F}$ ) are approximately reproduced by this simplified procedure. The measured values from this combination are $\kappa_{V}=1.026_{-0.044}^{+0.042}$ and $\kappa_{F}=0.88_{-0.09}^{+0.10}$.

The electroweak fit results in $\kappa_{V}=1.037_{-0.026}^{+0.029}$, $1.027_{-0.019}^{+0.020}$ and $1.021_{-0.014}^{+0.015}$, for cut-off parameters $\lambda=1$, 3 and $10 \mathrm{TeV}$, respectively, where $\lambda$ has been fixed during each of the fits. Including constraints from electroweak precision observables, the constraint on $\kappa_{V}$ can be improved by a factor of more than 3 . There is a mild dependence-both in the central value and uncertainty-on the chosen value for $\lambda$, but all values result in small but positive deviations from unity. For $\kappa_{V} \sim 1.03$ and $\lambda=4 \pi v$, the new physics scale is $\Lambda \gtrsim 13 \mathrm{TeV}$.

The slight positive deviation of $\kappa_{V}$ from 1 is driven by the small discrepancy between the observed and predicted values of the $W$ mass, as shown in Fig. 9 (top). The figure exhibits the strong correlation between the two quantities, and also the dependence on the chosen value of $\lambda$. To determine the predicted ellipses, the measured value of $M_{W}$ and the current measurements of $\mu_{i}$ have been removed from the EW fit.

Figure 9 (bottom) shows the prospects for predicting and measuring $\kappa_{V}$ versus $M_{W}$ at the LHC and ILC/GigaZ. For LHC, the predicted precision on $\kappa_{V}$ is largely limited by theoretical uncertainties somewhat optimistically set to $3 \%$ $[73,74]$. For the ILC, the predicted uncertainties on the measurements of the Higgs to $W$ and $Z$ gauge boson coupling
Table 5 The ATLAS and CMS Higgs coupling measurements of $\mu_{\mathrm{ggF}+\mathrm{ttH}}$ and $\mu_{\mathrm{VBF}+\mathrm{VH}}$ and their correlations, as used in this study. Unless where available, the central values, uncertainties and correlations have been estimated from published or public likelihood iso-contour lines

\begin{tabular}{|c|c|c|c|c|c|}
\hline Experiment & Channel & $\mu_{\mathrm{ggF}+\mathrm{ttH}}$ & $\mu_{\mathrm{VBF}+\mathrm{VH}}$ & Correlation & Refs \\
\hline \multirow[t]{3}{*}{ ATLAS } & $H \rightarrow \gamma \gamma, W W^{\star}, Z Z^{\star}$ & \multicolumn{3}{|c|}{ Published 2D-likelihood scan } & {$[57]$} \\
\hline & $H \rightarrow \gamma \gamma$ & $1.13_{-0.31}^{+0.37}$ & $1.15_{-0.58}^{+0.63}$ & -0.45 & [72] \\
\hline & $H \rightarrow W W^{\star}$ & $0.70_{-0.20}^{+0.25}$ & $0.70_{-0.50}^{+0.65}$ & -0.26 & [61] \\
\hline \multirow[t]{3}{*}{ CMS } & $H \rightarrow Z Z^{\star}$ & $0.80_{-0.36}^{+0.46}$ & $1.70_{-2.10}^{+2.20}$ & -0.75 & {$[62]$} \\
\hline & $H \rightarrow \tau \tau$ & $0.50_{-0.53}^{+0.53}$ & $1.30_{-0.40}^{+0.46}$ & -0.40 & {$[63]$} \\
\hline & $H \rightarrow b b$ & - & $1.00_{-0.50}^{+0.50}$ & - & [64] \\
\hline
\end{tabular}



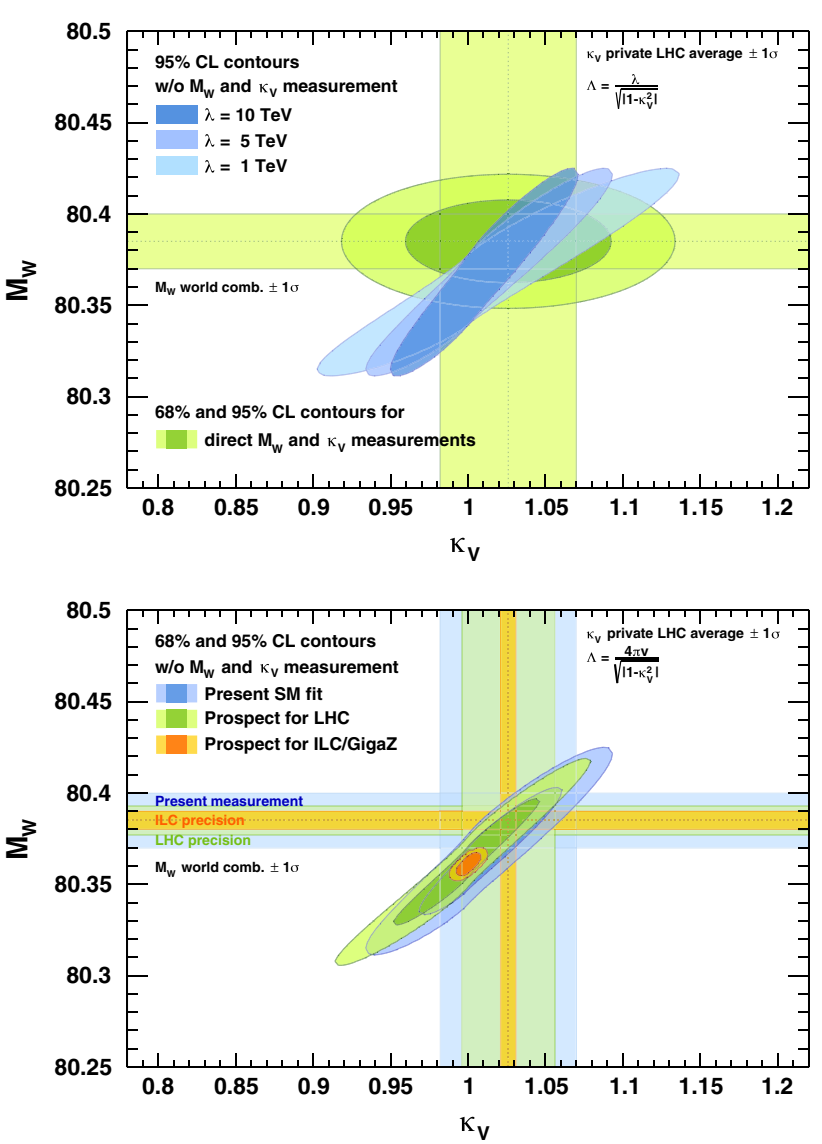

Fig. 9 Top Comparison of the direct $M_{W}$ and $\kappa_{V}$ measurements (horizontal and vertical green bands) with the contours of $95 \%$ CL allowed regions obtained from global fits for various values of the cut-off scale $\lambda$, in which the direct measurements of $M_{W}$ and $\kappa_{V}$ are not included. Bottom Similar comparison of the direct $M_{W}$ and $\kappa_{V}$ measurements and their indirect predictions for $\lambda=3 \mathrm{TeV}$, for the present (blue) and the ILC/GigaZ (yellow/orange) precision, at 68 and $95 \%$ CL. For better visibility the experimental ellipse is not drawn in the lower plot

constants are both $1 \%$ [75]. Assuming custodial symmetry, these uncertainties have been averaged in the figure. For the indirect LHC and ILC predictions, the central values of the electroweak observables have been shifted to match the Higgs mass of $125 \mathrm{GeV}$, with $\kappa_{V}=1$. The nominal value of $\lambda$ is $3 \mathrm{TeV}$. Varying $\lambda$ between 1,3 and $10 \mathrm{TeV}$, the central value of $\kappa_{V}$ remains unchanged at 1 , but its uncertainty varies between 0.008 and 0.015 at the LHC and between 0.003 and 0.005 for the ILC scenario. The numbers obtained for $\lambda=3 \mathrm{TeV}$ are summarised in Table 3. Assuming the present central values of $\kappa_{V}$ and $M_{W}$, the deviation of $\kappa_{V}$ from 1 would become significant.

\section{Conclusion}

We have updated in this paper the results from the global electroweak fit using full fermionic two-loop calculations for the partial widths and branching ratios of the $Z$ boson [10] and including a detailed assessment of the impact of theoretical uncertainties. The prospects of the fit in view of future colliders, namely the Phase-1 LHC and the ILC with GigaZ mode, were also studied. A significant increase in the predictive power of the fit was found in both scenarios, where in particular the ILC/GigaZ provides excellent sensitivity to indirect new physics. We have also carried out an analysis of the Higgs coupling data in a benchmark model with modified effective SM Higgs couplings to fermions and bosons parametrised by one parameter each. The inclusion of electroweak precision observables yields constraints on the bosonic coupling $\kappa_{V}$ that are about twice stronger than current Higgs coupling data alone, while the precision on the fermionic coupling $\kappa_{F}$ is not improved.

Acknowledgments We thank Ayres Freitas for intensive discussions, help concerning the electroweak calculations and a careful reading of this manuscript. This work is supported by the German Research Foundation (DFG) in the Collaborative Research Centre (SFB) 676 "Particles, Strings and the Early Universe" located in Hamburg.

Open Access This article is distributed under the terms of the Creative Commons Attribution License which permits any use, distribution, and reproduction in any medium, provided the original author(s) and the source are credited.

Funded by $\mathrm{SCOAP}^{3}$ / License Version CC BY 4.0.

\section{References}

1. ALEPH, DELPHI, L3, OPAL, LEP Electroweak, S. Schael et al., Phys. Rep. 532, 119 (2013). 1302.3415

2. J. Erler, Phys. Rev. D 81, 051301 (2010). 1002.1320

3. H. Flacher et al., Eur. Phys. J. C 60, 543 (2009), 0811.0009 [Erratum-ibid. C 71, 1718 (2011)]

4. M. Baak et al., Eur. Phys. J. C 72, 2003 (2012). 1107.0975

5. M. Ciuchini, E. Franco, S. Mishima, L. Silvestrini, JHEP 1308, 106 (2013). 1306.4644

6. ATLAS Collaboration, Phys. Lett. B (2012). 1207.7214

7. CMS Collaboration, Phys. Lett. B (2012). 1207.7235

8. M. Baak et al., Eur. Phys. J. C 72, 2205 (2012). 1209.2716

9. O. Eberhardt et al., Phys. Rev. Lett. 109, 241802 (2012). 1209.1101

10. A. Freitas, JHEP 1404, 070 (2014). 1401.2447

11. H. Baer et al., 1306.6352

12. The ALEPH, DELPHI, L3, OPAL, SLD Collaborations, the LEP Electroweak Working Group, the SLD Electroweak and Heavy Flavour Working Groups, Phys. Rep. 427, 257 (2006). hep-ex/0509008

13. M. Awramik, M. Czakon, A. Freitas, G. Weiglein, Phys. Rev. Lett. 93, 201805 (2004). hep-ph/0407317

14. M. Awramik, M. Czakon, A. Freitas, JHEP 0611, 048 (2006). hep-ph/0608099

15. L. Avdeev, J. Fleischer, S. Mikhailov, O. Tarasov, Phys. Lett. B 336, 560 (1994). hep-ph/9406363 [Erratum-ibid. B 349, 597 (1994)]

16. K. Chetyrkin, J.H. Kühn, M. Steinhauser, Phys. Lett. B 351, 331 (1995). hep-ph/9502291

17. K. Chetyrkin, J.H. Kühn, M. Steinhauser, Phys. Rev. Lett. 75, 3394 (1995). hep-ph/9504413

18. J. van der Bij, K. Chetyrkin, M. Faisst, G. Jikia, T. Seidensticker, Phys. Lett. B 498, 156 (2001). hep-ph/0011373 
19. M. Faisst, J.H. Kühn, T. Seidensticker, O. Veretin, Nucl. Phys. B 665, 649 (2003). hep-ph/0302275

20. Y. Schröder, M. Steinhauser, Phys. Lett. B 622, 124 (2005). hep-ph/0504055

21. K.G. Chetyrkin, M. Faisst, J.H. Kühn, P. Maierhofer, C. Sturm, Phys. Rev. Lett. 97, 102003 (2006). hep-ph/0605201

22. R. Boughezal, M. Czakon, Nucl. Phys. B 755, 221 (2006). hep-ph/0606232

23. M. Awramik, M. Czakon, A. Freitas, B. Kniehl, Nucl. Phys. B 813, 174 (2009). 0811.1364

24. M. Awramik, M. Czakon, A. Freitas, G. Weiglein, Phys. Rev. D 69, 053006 (2004). hep-ph/0311148

25. A. Freitas, Phys. Lett. B 730, 50 (2014). 1310.2256

26. A. Freitas, Y.-C. Huang, JHEP 1208, 050 (2012). 1205.0299 [Erratum-ibid. 1305, 074 (2013), Erratum-ibid. 1310, 044 (2013)]

27. K. Chetyrkin, J.H. Kuhn, A. Kwiatkowski, Phys. Rep. 277, 189 (1996)

28. P. Baikov, K. Chetyrkin, J.H. Kuhn, Phys. Rev. Lett. 101, 012002 (2008). 0801.1821

29. P. Baikov, K. Chetyrkin, J. Kuhn, J. Rittinger, Phys. Rev. Lett. 108, 222003 (2012). 1201.5804

30. A. Kataev, Phys. Lett. B 287, 209 (1992)

31. A. Czarnecki, J.H. Kühn, Phys. Rev. Lett. 77, 3955 (1996). hep-ph/9608366

32. R. Harlander, T. Seidensticker, M. Steinhauser, Phys. Lett. B 426, 125 (1998). hep-ph/9712228

33. G.-C. Cho, K. Hagiwara, Y. Matsumoto, D. Nomura, JHEP 1111, 068 (2011). 1104.1769

34. A.H. Hoang, A. Jain, I. Scimemi, I.W. Stewart, Phys. Rev. Lett. 101, 151602 (2008). 0803.4214

35. A.H. Hoang, I.W. Stewart, Nucl. Phys. Proc. Suppl. 185, 220 (2008). 0808.0222

36. A. Buckley et al., Phys. Rep. 504, 145 (2011). 1101.2599

37. S. Moch et al., 1405.4781

38. P. Skands, D. Wicke, Eur. Phys. J. C 52, 133 (2007). hep-ph/0703081

39. D. Wicke, P.Z. Skands, Nuovo Cim. B 123, S1 (2008). hep-ph/0807.3248

40. K. Chetyrkin, M. Steinhauser, Nucl. Phys. B 573, 617 (2000). hep-ph/9911434

41. K. Melnikov, T.v. Ritbergen, Phys. Lett. B 482, 99 (2000). hep-ph/9912391

42. M. Mangano, Private communication, Jun 2014. See also presentation at TOP2012 Interpreting the top quark mass: theoretical and MC aspects, Nov 2012
43. CMS Collaboration, CMS-PAS-TOP-12-029 (2012)

44. A. Hoang et al., Eur. Phys. J. Direct C 2, 1 (2000). hep-ph/0001286

45. Particle Data Group, J. Beringer et al., Phys. Rev. D 86, 010001 (2012)

46. M. Davier, A. Hoecker, B. Malaescu, Z. Zhang, Eur. Phys. J. C 71, 1515 (2011). 1010.4180

47. ATLAS Collaboration, CDF Collaboration, CMS Collaboration, D0 Collaboration. 1403.4427

48. ATLAS Collaboration, 1406.3827

49. CMS Collaboration, CMS-PAS-HIG-14-009 (2014)

50. M.E. Peskin, T. Takeuchi, Phys. Rev. Lett. 65, 964 (1990)

51. M.E. Peskin, T. Takeuchi, Phys. Rev. D 46, 381 (1992)

52. G. Altarelli, R. Barbieri, Phys. Lett. B 253, 161 (1991)

53. G. Altarelli, R. Barbieri, S. Jadach, Nucl. Phys. B 369, 3 (1992) [Erratum-ibid. B 376, 444 (1992)]

54. G. Bozzi, J. Rojo, A. Vicini, Phys. Rev. D 83, 113008 (2011). 1104.2056

55. R. Hawkings, K. Mönig, Eur. Phys. J. Direct C 1, 8 (1999). hep-ex/9910022

56. M. Davier, Private communication, Nov 2012

57. ATLAS Collaboration, Phys. Lett. B (2013). 1307.1427

58. ATLAS Collaboration, ATLAS-CONF-2013-108 (2013)

59. ATLAS Collaboration, ATLAS-CONF-2013-079 (2013)

60. CMS Collaboration, CMS-PAS-HIG-13-005 (2013)

61. CMS Collaboration, JHEP 1401, 096 (2014). 1312.1129

62. CMS Collaboration, Phys. Rev. D 89, 092007 (2014). 1312.5353

63. CMS Collaboration, JHEP 1405, 104 (2014). 1401.5041

64. CMS Collaboration, Phys. Rev. D 89, 012003 (2014). 1310.3687

65. LHC Higgs Cross Section Working Group, A. David et al., 1209.0040

66. J. Espinosa, C. Grojean, M. Muhlleitner, M. Trott, JHEP 1212, 045 (2012). 1207.1717

67. M. Farina, C. Grojean, E. Salvioni, JHEP 1207, 012 (2012). 1205.0011

68. B. Batell, S. Gori, L.-T. Wang, JHEP 1301, 139 (2013). 1209.6382

69. T. Corbett, O. Eboli, J. Gonzalez-Fraile, M. Gonzalez-Garcia, Phys. Rev. D 87, 015022 (2013). 1211.4580

70. A. Falkowski, F. Riva, A. Urbano, JHEP 1311, 111 (2013). 1303.1812

71. B. Dumont, S. Fichet, G. von Gersdorff, JHEP 1307, 065 (2013). 1304.3369

72. CMS Collaboration, 1407.0558

73. ATLAS Collaboration, ATL-PHYS-PUB-2013-007 (2013)

74. CMS Collaboration, CMS-NOTE-2012-006 (2012)

75. M.E. Peskin, 1207.2516 